\section{An Alternate Algorithm for Discrete-Time Filtering}

FREDERICK H. ZEITZ, III

PETER S. MAYBECK

Air Force Institute of Technology

The discrete-time Kalman filter is an optimal estimator for the states of a linear, stochastic system. It assumes that measurements are linear combinations of the states, and all disturbances are Gaussian. The influence diagram is a decision analysis tool. When the influence diagram represents Gaussian random variables, it provides an algorithm for discrete-time filtering equivalent to the Kalman filter. The influence diagram algorithm is a factored form of the Kalman filter, similar to other factored forms such as the U-D filter. Compared with the Kalman filter, it offers improved numerical properties. Compared with other factored forms, it offers a reduced computational load.
Manuscript received June 11, 1991; revised August 25, 1992.

IEEE Log No. T-AES/29/4/10989.

Authors' address: Department of Electrical and Computer Engineering, Air Force Institute of Technology, 2950 P Street, Wright-Patterson AFB, OH 45433-7765.

U.S. Government work not protected by U.S. copyright.

\section{INTRODUCTION}

The discrete-time Kalman filter is an optimal estimator for the states of a linear, stochastic system. It can be derived by assuming that the state estimates can be expressed as jointly Gaussian random variables. Using linear operations and Bayes' rule, the estimate of the state at any time can be calculated as a vector of conditional means and a conditional covariance matrix, conditioned on current measurements and the previous state estimate (using sufficient statistics).

It has been shown that, under some circumstances, the Kalman filter can have unsatisfactory numeric accuracy when implemented on a digital computer $[2,3,4,7]$. These accuracy problems are due to fixed computer wordlength and roundoff error. These errors can cause filter divergence or computations that are so erroneous as to be meaningless. This type of error may be manifested as a covariance matrix computed erroneously as having negative eigenvalues.

One reliable solution is to use alternative algorithms that have better numerical properties than Kalman's original equations. One class of algorithms that yields satisfactory results uses a square root factorization of the covariance matrix [5]. Square root factorizations generally have less dynamic range associated with their calculations, and are less susceptible to roundoff error. Another advantage of the square root representation is that the covariance matrix does not need to be calculated directly. Instead, it is represented in terms of its square root matrices. This factored form cannot represent a matrix with negative eigenvalues.

There are some disadvantages to factored forms of the Kalman filter. One significant disadvantage is that they normally require more computations, and therefore more computer time, than the conventional Kalman filter. The Potter covariance square root, the Carlson covariance square root, and the U-D filter are three of the more common factorization methods for discrete-time Kalman filtering. An explanation of each of these methods, along with a discussion of their numeric properties and a comparison of their speed, is given in Maybeck [7].

Of these factored forms, the U-D filter offers a high degree of numerical precision along with a reasonable increase in the required number of mathematical operations. Because of these properties, the U-D filter is a standard for comparison in the remainder of this work. The U-D filter is a factorization of the covariance matrix in the form $\mathbf{U D U}^{\mathrm{T}}$, where $\mathrm{U}$ is an upper triangular unit matrix (it has ones along the main diagonal) and $\mathbf{D}$ is a diagonal matrix. A thorough analysis of the U-D filter is presented in several works by Bierman and a case study by Bierman and Thornton [2-4].

The influence diagram is a graphical decision analysis tool that shows how random variables and 
decisions affect (or influence) a value function $[6,9,10,11]$. The influence diagram can be used for probabilistic analysis by using only random and deterministic variables (a deterministic variable is one that is precisely determined by the realizations of other variables). Under these conditions, the influence diagram represents a joint distribution function of the variables. The joint distribution is represented in the form of either a conditional or an unconditional distribution for each variable. One variable is given in unconditional form, a second variable is conditioned on the first, a third is conditioned on the first two, and so on. Each variable is a node in the influence diagram, with random variables represented pictorially as circles and deterministic variables represented as double circles. The conditioning relationships are represented as arrows from the conditioning variables to the variables being conditioned. The lack of an arrow between two nodes represents independence. If a random variable is conditioned on another, then the conditioning order can be reversed with Bayes' rule. In this manner, any random variable can be expressed in unconditional form, or as being conditioned on any other subset of random variables.

When the random variables are continuous and jointly Gaussian then a conditional Gaussian distribution is completely specified by the conditional mean, the conditional variance, and the effects of conditioning variables. In the influence diagram, the effects of conditioning variables are represented as the linear changes in the conditional means, given the realizations of the conditioning variables. These linear changes are the regression coefficients between the conditioned and the conditioning variables, identical to Yule's partial regression coefficients for multiple variables $[6,15]$.

The operations of a linear stochastic system can also be expressed in influence diagram form. The states of the system at a given time can be represented as conditional random variables, given the states at a previous time. Similarly, measurements can be represented as conditional random variables, given the present states. Bayes' rule can be used to reverse the conditioning of individual nodes, allowing the calculation of the conditional mean and variance of the present states, given the previous state estimate and the current measurement. Thus, the influence diagram affords an alternative algorithm for discrete-time filtering. Previous research [6] proved the feasibility of using the influence diagram algorithm for discrete-time filtering. It also demonstrated that the number and type of mathematical operations are essentially the same as required by the U-D filter.

The current research [16] investigates the computational loading and numerical properties of the influence diagram algorithm as applied to discrete-time filtering. This research demonstrates three improvements to the original algorithm that significantly decrease the number of operations required. This research also investigates the numerical properties of the influence diagram by relating them to known matrix operations: matrix operations with properties that are well understood. While previous research showed that the influence diagram algorithm represents a factored form of the covariance matrix $[6,11]$, the current research demonstrates that the numerical properties of the influence diagram are very similar to those of the U-D filter.

Because the influence diagram is a relatively new tool, it is assumed that most readers are unfamiliar with it. For this reason, the initial part of this paper is a tutorial based upon previous research $[6,9,10$, 11]. It explains the influence diagram algorithm and its application to discrete-time filtering.

\section{THE INFLUENCE DIAGRAM}

The influence diagram can be thought of as a pictorial representation of a joint distribution of random variables. Assume two random variables, $x$ and $y$ with a joint probability density function of $f_{x, y}(\xi, \rho)$ where $\xi$ and $\rho$ are dummy variables representing the possible realizations of the random variables. Either random variable can be represented as a marginal probability density function as $f_{x}(\xi)$ or $f_{y}(\rho)$. If the joint probability density function is given, then the marginal density function can be calculated by integrating the joint function over all possible realizations of the other random variable. For example, the equation for calulating the marginal density function of $y$ is

$$
f_{y}(\rho)=\int_{-\infty}^{\infty} f_{x, y}(\xi, \rho) d \xi .
$$

Bayes' rule can be used to calculate a conditional density function by

$$
f_{x \mid y}(\xi \mid \rho)=\frac{f_{x, y}(\xi, \rho)}{f_{y}(\rho)}
$$

where $f_{x \mid y}(\xi \mid \rho)$ is the conditional density function of $x$ given $y$. The conditional density function $f_{y \mid x}(\rho \mid \xi)$ can be calculated in a similar manner by

$$
f_{y \mid x}(\rho \mid \xi)=\frac{f_{x, y}(\xi, \rho)}{f_{x}(\xi)}
$$

Any joint probability density function can be expressed, using Bayes' rule, as a product of conditional and marginal density functions. For example, the joint probability density function of three random variables $x, y$, and $z$ can be written as

$$
f_{x, y, z}(\xi, \rho, \zeta)=f_{x}(\xi) f_{y \mid x}(\rho \mid \xi) f_{z \mid x, y}(\zeta \mid \xi, \rho) .
$$

An influence diagram represents the joint probability density function in terms of the conditional forms in (4). For this example, the influence diagram is 


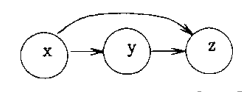

Fig. 1. Influence diagram for 3 variables.

shown in Fig. 1. Each random variable is represented as a circle. If any variable were deterministic then it would be depicted as a double circle. The conditional relationships are shown by the arrows between the circles.

Nodes in an influence diagram that have arrows pointing to another node are called the conditional or direct predecessors of that node. The node at the end of the arrow is called the successor node. The nodes in an influence diagram must be "ordered", meaning that a node's predecessors cannot also be its successors, either directly or indirectly through other nodes. If a sequence of nodes cannot be ordered, then a cycle is said to exist. A cycle implies circular conditioning, which is not allowed.

Transformations can change the appearance of the influence diagram and affect the conditional distributions underlying the diagram. The two essential transformations are arc reversal and node removal. Arc reversal is the process of reversing the position of two nodes in an ordered sequence. For example, nodes $x$ and $y$ can be reversed in Fig. 1 . This requires the calculation of the marginal (or unconditional) density function of $y$ and the conditional density function of $x$ given $y$. Using Bayes' rule and the integral in (1) the specific equations are

$$
\begin{aligned}
f_{y}(\rho) & =\int_{-\infty}^{\infty} f_{x}(\xi) f_{y \mid x}(\rho \mid \xi) d \xi \\
f_{x \mid y}(\xi \mid \rho) & =\frac{f_{x}(\xi) f_{y \mid x}(\rho \mid \xi)}{f_{y}(\rho)} .
\end{aligned}
$$

The mathematics in (5) and ' explicitly, but are implied by the diagram.

To preserve an ordered list and prevent a cycle, a pair of nodes can only be reversed when they may be placed next to each other in some ordered sequence. Arc reversal also implies that each node of the pair inherits the predecessors of the other node in addition to its own. If there is no arc between two adjacent nodes, then those nodes are independent, given their conditional predecessors. In this case, the order may be reversed with no calculations required. If, after an arc reversal, the successor node has no other successors (no variables that it conditions), and if it has no further use in the influence diagram, then it can be removed from the diagram as a "nuisance" variable. The remaining influence diagram represents the joint distribution of the remaining variables.

The influence diagram can be used for either continuous or discrete random variables. It does not show the actual density functions; it is merely a pictorial method of representing the conditional relationships between the variables and how they may be changed.

If all random variables in an influence diagram are jointly Gaussian, then they can be considered as elements of a Gaussian random vector. Each node in the Gaussian influence diagram represents one of the random (or deterministic) variables and the entire influence diagram represents the joint density function of the random vector. A deterministic linear combination of jointly Gaussian random variables is also Gaussian. In this case, deterministic variables can be considered as a degenerate form of a Gaussian random variable: random variables that have a variance of zero. The mathematics for transforming a Gaussian influence diagram (using node reversal or removal) are simpler than for the general case because of the convenient properties of a Gaussian conditional variable $[1,7,8]$.

Assume an example of two jointly Gaussian random variables $x$ and $y$ making up a random vector. The influence diagram expresses the joint density as the mean and variance of one variable, along with the conditional mean and conditional variance of the second, given the first. This conditional density is Gaussian because any conditional density of jointly Gaussian random variables is Gaussian also. The two Gaussian random variables are $x$ and $y$. The mean vector and covariance matrix are

$$
\begin{aligned}
& \mathbf{m}=\left[\begin{array}{l}
\mu_{x} \\
\mu_{y}
\end{array}\right] \\
& \mathbf{P}=\left[\begin{array}{ll}
P_{x x} & P_{x y} \\
P_{y x} & P_{y y}
\end{array}\right]
\end{aligned}
$$

where $\mu_{x}$ and $\mu_{y}$ are the unconditional means of $x$ and $y$, respectively. The notation $P_{x y}$ implies the covariance of the variables $x$ and $y$ and is equal to $P_{y x} . P_{x x}$ represents the covariance of variable $x$ with itself, or just the variance of $x$.

The conditional mean of $x$ given $y$ can be calculated from the mean vector and the covariance matrix [7, pp. 110-111]. The conditional mean is

$$
\begin{aligned}
& \mu_{x \mid y}=\mu_{x}+\frac{P_{x y}}{P_{y y}}\left(\rho-\mu_{y}\right) \\
& \mu_{x \mid y}=\mu_{x}+b_{y x}\left(\rho-\mu_{y}\right)
\end{aligned}
$$

where $\rho$ is a dummy variable representing the possible realizations of the variable $y$. The quantity in parenthesis is the difference of predicted (mean) value of the random variable and its realization. This term will be called the residual. The conditional variance of $x$ given $y$ is

$$
\begin{aligned}
& P_{x \mid y}=P_{x x}-\frac{P_{x y}^{2}}{P_{y y}} \\
& P_{x \mid y}=P_{x x}-P_{x y} b_{y x} .
\end{aligned}
$$




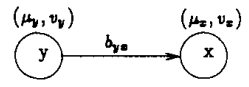

Fig. 2. Influence diagram for continuous Gaussian random variables.

The term $b_{y x}$ appears in both equations. It is called the regression coefficient of $x$ on $y$ and it represents a linear change in the conditional mean of $x$, given the realization of $y$. The regression coefficient is not the same as the correlation coefficient $r_{x y}$, but it is related by the expression

$$
b_{y x}=r_{x y} \frac{\sqrt{P_{x x}}}{\sqrt{P_{y y}}} .
$$

If the order of conditioning were reversed, then the conditional mean of $y$ given $x$ would be

$$
\begin{aligned}
& \mu_{y \mid x}=\mu_{y}+\frac{P_{y x}}{P_{x x}}\left(\xi-\mu_{x}\right) \\
& \mu_{y \mid x}=\mu_{y}+b_{x y}\left(\xi-\mu_{x}\right)
\end{aligned}
$$

where $\xi$ is now the dummy variable representing the possible realizations of the variable $x$. The conditional variance of $y$ given $x$ is

$$
\begin{aligned}
& P_{y \mid x}=P_{y y}-\frac{P_{y x}^{2}}{P_{x x}} \\
& P_{y \mid x}=P_{y y}-P_{y x} b_{x y} .
\end{aligned}
$$

The term $b_{x y}$ is the regression coefficient of $y$ on $x$. It too is related to the correlation coefficient $r_{x y}$, this time by the relationship

$$
b_{x y}=r_{x y} \frac{\sqrt{P_{y y}}}{\sqrt{P_{x x}}} .
$$

The order of the subscripts in $b_{y x}$ and $b_{x y}$ is significant because $b_{y x} \neq b_{x y}$ in general.

Assume a Gaussian influence diagram and its underlying density as shown in Fig. 2. In this case, the random variable $y$ is given in unconditional form, and the random variable $x$ is expressed in conditional form. Only five values are sufficient to specify the joint distribution: the unconditional means of the two random variables, the unconditional variance of $y$, the conditional variance of $x$, and the regression coefficient of $x$ on $y$. These five values are shown directly on the influence diagram. In this figure, $v_{i}$ is the variance of the $i$ th node, whether it is in conditional or unconditional form. The means and variances are associated with the appropriate node, while the regression coefficients are associated with the arrow between the nodes.

The arrow between the nodes can be reversed as in the general case, and the joint density function expressed as an unconditional density of $x$ and a

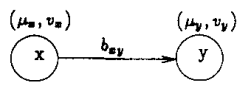

Fig. 3. Continuous Gaussian influence diagram after reversal.

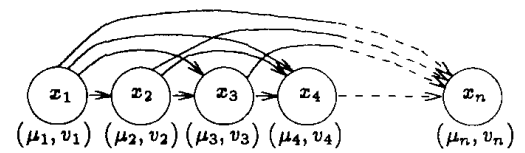

Fig. 4. Influence diagram for $n$ jointly Gaussian random variables.

conditional density of $y$. This is shown in the influence diagram in Fig. 3. The equations for changing the order are given later.

If there are $n$ random variables in the Gaussian random vector, then one is chosen, perhaps arbitrarily, to be represented in unconditional form. A second random variable is conditioned on the first as in the previous example. The third random variable is represented as being conditioned on the first two. There will be two regression coefficients associated with this third variable, one from the first variable and one from the second. The process continues with the fourth variable conditioned on the previous three and having three regression coefficients, etc. Eventually, the $n$th random variable is represented as a conditional density, conditioned on the previous $n-1$ variables, with a regression coefficient associated with each. The result can be written in the form of an $n$-dimensional influence diagram. Using simplified notation where $f_{x_{n}}$ implies $f_{x_{n}}\left(\xi_{n}\right)$, the diagram shown in Fig. 4 can be expressed mathematically as

$$
f_{x_{1}, x_{2}, x_{3} \ldots x_{n}}=f_{x_{1}} f_{x_{2} \mid x_{1}} f_{x_{3} \mid x_{1}, x_{2}} \ldots f_{x_{n} \mid x_{1}, x_{2}, x_{3} \ldots x_{n-1}} .
$$

For legibility, Fig. 4 and all subsequent influence diagrams may not show the means, variances, or regression coefficients even though their existence is implied. As stated previously, adjacent nodes are conditionally independent if no arc exists between them. In the Gaussian influence diagram, a missing arc corresponds to a regression coefficient of zero.

Any two nodes in an influence diagram can be reversed if they can be placed next to each other in some ordered sequence. Call the first variable $x_{i}$ and the second $x_{j}$ where it is assumed that $x_{i}$ is a conditional predecessor of $x_{j}$. Furthermore, assume that $\left\{x_{K}\right\}$ is a set containing the union of all direct predecessor nodes of both $x_{i}$ and $x_{j}$, except for node $i$ itself. Also assume that $x_{k}$ is an arbitrary element of $\left\{x_{K}\right\}$. Nodes $x_{i}$ and $x_{j}$ have variance $v_{i}$ and $v_{j}$, respectively. The term $b_{i j}$ is the regression coefficient of $j$ on $i$; the terms $b_{k i}$ and $b_{k j}$ are the regression coefficients of $x_{i}$ and $x_{j}$ on $x_{k}$.

After reversal of the two nodes, both nodes will inherit each others' direct predecessors. Furthermore, 
the regression coefficients from predecessor nodes must be adjusted to reflect the new conditioning order. The equations for calculating the new variances and regression coefficients are as follows, where the prime symbols represent a new value. For node $j$, no longer conditioned on node $i$, the new variance and regression coefficients from predecessors are

$$
\begin{gathered}
v_{j}^{\prime}=v_{j}+b_{i j}^{2} v_{i} \\
b_{k j}^{\prime}=b_{k j}+b_{k i} b_{i j} .
\end{gathered}
$$

For node $i$, conditioned on node $j$, the new variance and regression coefficients are

$$
\begin{aligned}
v_{i}^{\prime} & =\frac{v_{i} v_{j}}{v_{j}^{\prime}} \\
b_{j i}^{\prime} & =\frac{v_{i} b_{i j}}{v_{j}^{\prime}} \\
b_{k i}^{\prime} & =b_{k i}-b_{k j}^{\prime} b_{j i}^{\prime} .
\end{aligned}
$$

The influence diagram represents the conditional mean in equation form similar to (10). That is, it represents the conditional mean of a variable as a linear function of the residual of predecessor variables. The random variable being realized is usually in unconditional form, meaning it has no visible predecessors. The realization of a random variable causes the conditional means of all successor nodes to change. Two terms, the "path product" and the "path coefficient" are introduced to explain the change of mean.

A path product is defined as the product of the regression coefficients on a given path from a predecessor node to a successor node. A path coefficient is defined as the sum of the path products on all possible paths from a predecessor node to a successor node. For example, an influence diagram of five nodes, numbered sequentially 1 through 5 , has path products from node 1 to node 5 of $b_{15}$, $b_{12} b_{25}, b_{13} b_{35}, b_{14} b_{45}, b_{12} b_{23} b_{35}, b_{12} b_{24} b_{45}, b_{13} b_{34} b_{45}$, $b_{12} b_{23} b_{34} b_{45}$. The sum of these path products results in the path coefficient $u_{15}$. In more general terms, let $u_{m, n}$ be the path coefficient from a node $m$ to a successor node $n$, and let $b_{m, n}$ be the regression coefficient on the arrow from a node $m$ to its direct successor node $n$. The expression for $u_{1, r}$ is

$$
\begin{aligned}
u_{1, r}= & b_{1, r}+b_{1, r-1}\left(u_{r-1, r}\right) \\
& +b_{1, r-2}\left(u_{r-2, r}\right)+\cdots+b_{1,2}\left(u_{2, r}\right) .
\end{aligned}
$$

The linear change in the conditional mean of a successor node, conditioned on the realization of a predecessor, is equal to the path coefficient from the first to the second. In equation form, the update of the $r$ th node based on the realization of the first node is

$$
\mu_{r \mid 1}=\mu_{r}+u_{1, r}\left(\zeta_{1}-\mu_{1}\right)
$$

where $\zeta_{1}$ is the realized value of the random variable associated with the first node. The process can be thought of as "propagating" the change in mean of the realized node to each successor node, via the respective path coefficient. In order to propagate the mean to all successor nodes, all path coefficients must be calculated. After a node in unconditional form is realized, it is no longer needed and can be removed from the influence diagram. Such a node is said to be "instantiated." The remaining successor nodes retain their conditioning on the instantiated node because their conditional means reflect the realized value of the instantiated node. In this manner, the nodes in an influence diagram can represent random variables that are conditioned on other random variables that have been removed from the diagram by instantiation.

\section{MATRIX REPRESENTATION OF THE INFLUENCE DIAGRAM}

The covariance matrix can be used to calculate the matrix of regression coefficients and the conditional variances for a random vector, similar to the simple scalar case presented in (10) and (12). The calculations are closely related to the Cholesky decomposition of the covariance matrix $[11,12,14]$. Let $\mathbf{P}$ be a positive definite, symmetric covariance matrix. A factorization of $\mathbf{P}$ exists in the form $\mathbf{P}=\mathbf{U}^{\mathrm{T}} \mathbf{S S U}$ where $\mathbf{U}$ is a unit upper triangular matrix and $\mathbf{S}$ is a diagonal matrix so that $\mathbf{S}^{\mathrm{T}}=\mathbf{S}$. The matrix $(\mathbf{S U})^{\mathrm{T}} \mathbf{S U}$ is identical to a lower triangular Cholesky decomposition of the covariance matrix $\mathbf{P}=\mathbf{L L}^{\mathrm{T}}$. The matrix $\mathbf{S S}=\mathbf{D}$ is also a diagonal matrix so the the covariance matrix can be represented as $\mathbf{U}^{\mathrm{T}} \mathrm{DU}$.

Let $I$ be the identity matrix, and $\mathbf{B}_{j}$ be a strictly upper triangular matrix that is all zeros except for the $j$ th column above the diagonal. The elements of the $\mathbf{B}_{j}$ matrix are the regression coefficients $b_{1 j}, b_{2 j}, \ldots, b_{j-1, j}$ where the subscripts indicate the predecessor and the successor node. The subscripts also correspond to the conventional row-column notation for matrix elements. The matrix $\mathbf{U}_{j}$ is a unit upper triangular matrix defined as $\left(\mathbf{I}-\mathbf{B}_{j}\right)$, and the matrix $\mathbf{B}$ is defined as $\mathbf{B}_{1}+\mathbf{B}_{2}+\mathbf{B}_{3}+\cdots+\mathbf{B}_{n}$ (where the convention leads to $\mathbf{B}_{1}=\mathbf{0}$ and $\mathbf{U}_{1}=\mathbf{I}$ ). Kenley and Schacter showed [11, pp. 547-548]:

$$
\mathrm{U}=\mathbf{U}_{1} \mathbf{U}_{2} \mathbf{U}_{3} \ldots \mathbf{U}_{n}
$$

and

$$
\mathbf{U}=(\mathbf{I}-\mathbf{B})^{-1} .
$$

The $\mathbf{D}$ and the $\mathbf{U}$ matrices can be computed by taking the Cholesky decomposition of the covariance matrix, then factoring the $U_{1}, U_{2}, U_{3}, \ldots, U_{n}$ matrices from the $\mathrm{U}$ matrix. A more efficient method is given in Kenley and Schacter [11, p. 549]. The $(i, j)$ element of the matrix $\mathbf{U}$ is the path coefficient $u_{i j}$. 


\section{THE INFLUENCE DIAGRAM FOR DISCRETE-TIME FILTERING}

If the variables in a Gaussian influence diagram represent the states of a linear system, then the influence diagram can be used as a conditional mean estimator for the states. Under these assumptions, it yields the same state estimates and variances as the Kalman filter [6, pp. 52-106].

Assume a linear system of the form:

$$
\mathbf{x}\left(t_{i}\right)=\mathbf{\Phi}\left(t_{i}, t_{i-1}\right) \mathbf{x}\left(t_{i-1}\right)+\mathbf{G}_{d}\left(t_{i-1}\right) \mathbf{w}_{d}\left(t_{i-1}\right)
$$

where $\mathbf{x}\left(t_{i}\right)$ and $\mathbf{x}\left(t_{i-1}\right)$ are $n$-dimensional state vectors at times $t_{i}$ and $t_{i-1}$ respectively. $\Phi\left(t_{i}, t_{i-1}\right)$ is the state transition matrix which describes the propagation of the states from time $t_{i-1}$ to time $t_{i}$. The term $\mathbf{w}_{d}\left(t_{i-1}\right)$ represents the process noise and is assumed to be a discrete-time zero-mean white Gaussian noise sequence with covariance kernel

$$
E\left\{\mathbf{w}_{d}\left(t_{i}\right) \mathbf{w}_{d}^{\mathrm{T}}\left(t_{j}\right)\right\}=\left\{\begin{array}{ll}
\mathbf{Q}_{d}\left(t_{i}\right), & t_{i}=t_{j} \\
\mathbf{0}, & t_{i} \neq t_{j}
\end{array} .\right.
$$

The matrix $\mathbf{G}_{d}\left(t_{i-1}\right)$ represents a linear operation which describes how the discrete-time noise enters the system at time $t_{i-1}$. The vectors $\mathbf{x}\left(t_{i-1}\right)$ and $\mathbf{w}_{d}\left(t_{i-1}\right)$ are assumed to be independent. The state vector at time $t_{i-1}$ is a Gaussian random vector with conditional mean, given the previous state estimates and measurements up to time $t_{i-1}$, defined as $\hat{\mathbf{x}}\left(t_{i-1}^{+}\right)$. The conditional covariance of the state estimate at time $t_{i-1}$ is $\mathbf{P}\left(t_{i-1}^{+}\right)$.

The conditional mean and covariance of the state vector at time $t_{i}$ can be calculated by the Kalman filter propagation equations:

$$
\begin{aligned}
\hat{\mathbf{x}}\left(t_{i}^{-}\right)= & \boldsymbol{\Phi}\left(t_{i}, t_{i-1}\right) \hat{\mathbf{x}}\left(t_{i-1}^{+}\right) \\
\mathbf{P}\left(t_{i}^{-}\right)= & \boldsymbol{\Phi}\left(t_{i}, t_{i-1}\right) \mathbf{P}\left(t_{i-1}^{+}\right) \mathbf{\Phi}^{\mathrm{T}}\left(t_{i}, t_{i-1}\right) \\
& +\mathbf{G}_{d}\left(t_{i-1}\right) \mathbf{Q}_{d}\left(t_{i-1}\right) \mathbf{G}_{d}^{\mathrm{T}}\left(t_{i-1}\right) .
\end{aligned}
$$

The $n$-dimensional estimate of the state vector at time $t_{i-1}$ can be expressed in influence diagram form as the leftmost column of $n$ random variable nodes in Fig. 5. The means of the nodes are the means of the vector $\hat{\mathbf{x}}\left(t_{i-1}^{+}\right)$. The regression coefficients and the conditional variances of the individual nodes are the factorization of the covariance matrix $\mathbf{P}\left(t_{i-1}^{+}\right)$. The influence diagram represents the conditional mean and variance of the state, conditioned on prior measurements. The prior measurements are not shown because they represent random variables that have been realized and instantiated (removed from the diagram).

The remainder of the influence diagram in Fig. 5 depicts the linear model of (29). The $r$-dimensional discrete-time zero-mean random noise vector $\mathbf{w}_{d}\left(t_{i-1}\right)$ is depicted as $r$ nodes in influence diagram form. The nodes of $\mathbf{w}_{d}\left(t_{i-1}\right)$ have no arrows drawn between

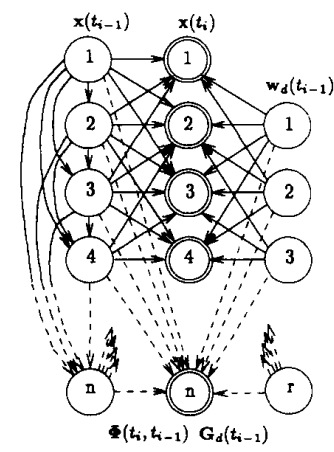

Fig. 5. Influence diagram depiction of $\mathbf{x}\left(t_{i}\right)=\boldsymbol{\Phi}\left(t_{i}, t_{i-1}\right) \mathbf{x}\left(t_{i-1}\right)+\mathbf{G}_{d}\left(t_{i-1}\right) \mathbf{w}_{d}\left(t_{i-1}\right)$

them, implying that the covariance matrix $\mathbf{Q}_{d}\left(t_{i-1}\right)$ is diagonal (this can be assumed without loss of generality, since $\mathbf{G}_{d}\left(t_{i-1}\right) \mathbf{Q}_{d}\left(t_{i-1}\right) \mathbf{G}_{d}^{\mathrm{T}}\left(t_{i-1}\right)$ can be factored using a U-D factorization, letting $\mathbf{G}_{d}\left(t_{i-1}\right)^{\prime}$ be the $U$-factor and $Q_{d}\left(t_{i-1}\right)^{\prime}$ be the $D$-factor). The mean of all nodes of $\mathbf{w}_{d}\left(t_{i-1}\right)$ is zero. The vector $\mathbf{x}\left(t_{i}\right)$ is depicted by a set of $n$ independent deterministic nodes. This is because it is a deterministic function of two independent Gaussian random vectors, $\mathbf{x}\left(t_{i-1}\right)$ and $\mathbf{w}_{d}\left(t_{i-1}\right)$. The linear relationship between $\mathbf{x}\left(t_{i}\right)$ and $\mathbf{x}\left(t_{i-1}\right)$ is represented by the regression coefficients on the arrows from the nodes of $\mathbf{x}\left(t_{i-1}\right)$ to the nodes of $\mathbf{x}\left(t_{i}\right)$. From (29), this linear relationship is the matrix $\Phi\left(t_{i}, t_{i-1}\right)$. Consequently, the regression coefficients on the arrows between the two vectors are the elements of the state transition matrix. The coefficient on the arrow between the $k$ th node of $\mathbf{x}\left(t_{i-1}\right)$ and the $j$ th node of $\mathbf{x}\left(t_{i}\right)$ corresponds to the $(j, k)$ element of $\boldsymbol{\Phi}\left(t_{i}, t_{i-1}\right) . \mathbf{Q}_{d}\left(t_{i-1}\right)$ is assumed to be diagonal and the linear operation corresponding to $\mathbf{G}_{d}\left(t_{i-1}\right)$ is depicted by arrows from the nodes of $\mathbf{w}_{d}\left(t_{i-1}\right)$ to $\mathbf{x}\left(t_{i}\right)$. The coefficient on the arrow from the $k$ th node of $\mathbf{w}_{d}\left(t_{i-1}\right)$ to the $j$ th node of $\mathbf{x}\left(t_{i}\right)$ is the $(j, k)$ element of $\mathbf{G}_{d}\left(t_{i-1}\right)$.

The influence diagram can be used to calculate the density function for $\mathbf{x}\left(t_{i}\right)$. Arc reversal is used to make each node of $\mathbf{w}_{d}\left(t_{i-1}\right)$ conditioned on the nodes of $\mathbf{x}\left(t_{i}\right)$. The nodes of $\mathbf{w}_{d}\left(t_{i-1}\right)$ can then be sequentially removed as "nuisance" variables. Similarly, each node of $\mathbf{x}\left(t_{i-1}\right)$ can be conditioned on the nodes of $\mathbf{x}\left(t_{i}\right)$ and removed. The most efficient method is to remove the highest numbered nodes first. The results of these operations are shown in Fig. 6. The regression coefficients and conditional variances of the nodes represent a factored form of $\mathbf{P}\left(t_{i}^{-}\right)$.

The Kalman filter assumes a measurement model that has measurements expressed as a linear combination of the system states with additive discrete-time Gaussian disturbances. In equation form, the model is

$$
\mathbf{z}\left(t_{i}\right)=\mathbf{H}\left(t_{i}\right) \mathbf{x}\left(t_{i}\right)+\mathbf{v}\left(t_{i}\right)
$$




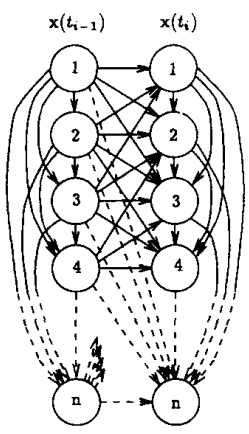

(a)

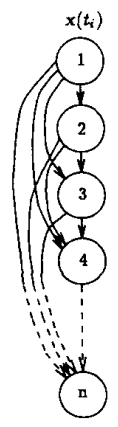

(b)

Fig. 6. Results of removing the vectors $\mathbf{w}_{d}\left(t_{i-1}\right)$ and $\mathbf{x}\left(t_{i-1}\right)$ (a) Influence diagram after $\mathbf{w}_{d}\left(t_{i-1}\right)$ removed. (b) Influence diagram after $\mathbf{x}\left(t_{i-1}\right)$ removed.

The term $\mathbf{H}\left(t_{i}\right)$ is the matrix which describes the linear combination of states that are available through the measuring devices. The term $\mathbf{v}\left(t_{i}\right)$ is the zero-mean discrete-time Gaussian noise with covariance kernel:

$$
E\left\{\mathbf{v}\left(t_{i}\right) \mathbf{v}^{\mathrm{T}}\left(t_{j}\right)\right\}=\left\{\begin{array}{ll}
\mathbf{R}\left(t_{i}\right), & t_{i}=t_{j} \\
\mathbf{0}, & t_{i} \neq t_{j}
\end{array} .\right.
$$

The state estimate at time $t_{i}$, given the previous state estimate and the measurements at time $t_{i}$, is a Gaussian random vector with conditional mean $\hat{\mathbf{x}}\left(t_{i}^{+}\right)$and conditional covariance matrix $\mathbf{P}\left(t_{i}^{+}\right)$. The optimum weighting of the measurement inputs, called the Kalman gain $\mathbf{K}\left(t_{i}\right)$, is also part of the calculations. The Kalman filter equations are

$$
\begin{aligned}
\mathbf{K}\left(t_{i}\right) & =\mathbf{P}\left(t_{i}^{-}\right) \mathbf{H}^{\mathrm{T}}\left(t_{i}\right)\left[\mathbf{H}\left(t_{i}\right) \mathbf{P}\left(t_{i}^{-}\right) \mathbf{H}^{\mathrm{T}}\left(t_{i}\right)+\mathbf{R}\left(t_{i}\right)\right]^{-1} \\
\hat{\mathbf{x}}\left(t_{i}^{+}\right) & =\hat{\mathbf{x}}\left(t_{i}^{-}\right)+\mathbf{K}\left(t_{i}\right)\left[\mathbf{z}_{i}-\mathbf{H}\left(t_{i}\right) \hat{\mathbf{x}}\left(t_{i}^{-}\right)\right] \\
\mathbf{P}\left(t_{i}^{+}\right) & =\mathbf{P}\left(t_{i}^{-}\right)-\mathbf{K}\left(t_{i}\right) \mathbf{H}\left(t_{i}\right) \mathbf{P}\left(t_{i}^{-}\right) .
\end{aligned}
$$

The influence diagram can depict the linear measurement model of (33). The $p$-dimensional measurement vector $\mathbf{z}\left(t_{i}\right)$ is a deterministic function of the $n$-dimensional random vector $\mathbf{x}\left(t_{i}\right)$ and the $p$-dimensional random vector $\mathbf{v}\left(t_{i}\right)$. Fig. 7

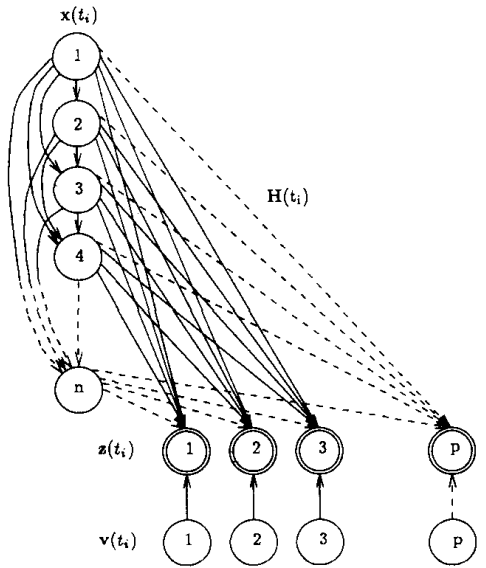

Fig. 7. Measurement update model for $\mathbf{z}\left(t_{i}\right)=\mathbf{H}\left(t_{i}\right) \mathbf{x}\left(t_{i}\right)+\mathbf{v}\left(t_{i}\right)$.

shows the influence diagram representation of this functional relationship. The vector $\mathbf{v}\left(t_{i}\right)$ is a zero-mean discrete-time Gaussian noise. The covariance matrix $\mathbf{R}\left(t_{i}\right)$ is represented in factored form by the variances of the nodes of $\mathbf{v}\left(t_{i}\right)$. The lack of arcs between the nodes of $\mathbf{v}\left(t_{i}\right)$ implies that $\mathbf{R}\left(t_{i}\right)$ is a diagonal matrix. The case of nondiagonal $\mathbf{R}\left(t_{i}\right)$ is addressed later. The measurement matrix $\mathbf{H}\left(t_{i}\right)$ is represented by the arrows from the nodes of $\mathbf{x}\left(t_{i}\right)$ to $\mathbf{z}\left(t_{i}\right)$. The regression coefficients on these arrows are the elements of $\mathbf{H}\left(t_{i}\right)$. They represent the linear combination of states that makes up the measurement vector. The arrows from the vector $\mathbf{v}\left(t_{i}\right)$ to the vector $\mathbf{z}\left(t_{i}\right)$ have regression coefficients of unity.

The desired density function is the vector $\mathbf{x}\left(t_{i}\right)$ conditioned on the vector $\mathbf{z}\left(t_{i}\right)$ (and previous measurements through the sufficient statistic $\hat{\mathbf{x}}\left(t_{i}^{-}\right)$). This means that the nodes of $\mathbf{z}\left(t_{i}\right)$ will be first in the ordered sequence, followed by the nodes of $\mathbf{x}\left(t_{i}\right)$. The first step is to remove the nodes of $\mathbf{v}\left(t_{i}\right)$. The arrows between $\mathbf{v}\left(t_{i}\right)$ and $\mathbf{z}\left(t_{i}\right)$ are reversed until each of the nodes of $\mathbf{v}\left(t_{i}\right)$ are conditioned on $\mathbf{z}\left(t_{i}\right)$ and removed as nuisance variables. If $\mathbf{R}\left(t_{i}\right)$ is diagonal as shown in Fig. 7, then each deterministic node of $\mathbf{z}\left(t_{i}\right)$ takes on the variance of the associated node of $\mathbf{v}\left(t_{i}\right)$. This operation is shown in the first influence diagram in Fig. 8. The second step in the process is to reverse the arcs between $\mathbf{z}\left(t_{i}\right)$ and $\mathbf{x}\left(t_{i}\right)$. The distribution of the vector $\mathbf{x}\left(t_{i}\right)$ is now conditioned on the vector $\mathbf{z}\left(t_{i}\right)$. These operations are shown in the second influence diagram in Fig. 8.

The linear operation depicted by the arrows from $\mathbf{z}\left(t_{i}\right)$ to $\mathbf{x}\left(t_{i}\right)$ in Fig. 8 represent the Kalman gain matrix. The path coefficient (not the regression coefficient) from node $k$ of $\mathbf{z}\left(t_{i}\right)$ to node $j$ of $\mathbf{x}\left(t_{i}\right)$ must be the $(j, k)$ element in the Kalman gain matrix.

The final calculation is to instantiate the nodes of $\mathbf{z}\left(t_{i}\right)$ in order. The realizations of these random variables are the measurements $\mathbf{z}\left(t_{i}\right)$. With each 


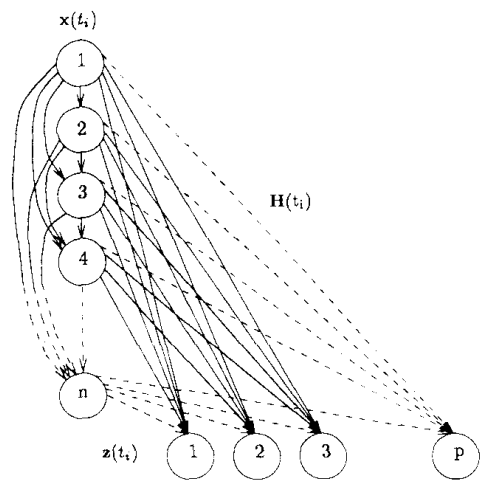

(a)

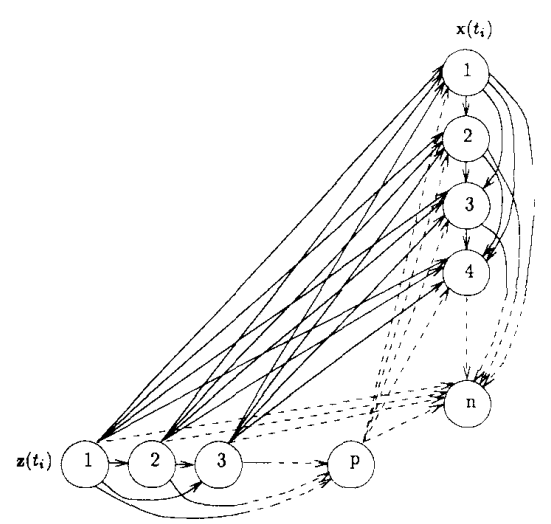

(b)

Fig. 8. Results of measurement update cycle. (a) After incorporating $\mathbf{v}\left(t_{i}\right)$. (b) After reversing conditioning of $\mathbf{x}\left(t_{i}\right)$ and $\mathbf{z}\left(t_{i}\right)$

realization (measurement), the difference between the mean and the realized value of the random variable (the residual) is propagated via the path coefficients to all subsequent nodes, and the instantiated node is removed. After all nodes of $\mathbf{z}\left(t_{i}\right)$ are removed, the remaining vector $\mathbf{x}\left(t_{i}\right)$ is conditioned on the measurements $\mathbf{z}\left(t_{i}\right)$ [6].

\section{IMPROVEMENTS TO THE INFLUENCE DIAGRAM ALCORITHM}

The previous discussion was a tutorial presentation of previous research in the influence diagram and its use for discrete-time filtering. The current research [16] offers three improvements to the previous influence diagram algorithm for discrete-time filtering. One improvement is a simpler method of updating the conditional means, the second improvement is a method for vector measurement updates, and the third improvement is a reduced number of mathematical operations under certain conditions. Each improvement is discussed separately.
Previous research proved that the Gaussian influence diagram was an alternative algorithm for discrete-time filtering, but it used the one-cycle model described in the previous tutorial [6]. In practice, each time interval requires the addition of another stage of influence diagrams. The existing algorithm for updating the change in conditional means at any time interval requires that the conditional mean must be updated when any measurement is made at a predecessor node [11, pp. 531-532]. Thus, a discrete-time filter for $n$ time intervals requires $n$ repetitions of the influence diagram. The conditional means at time $t_{n}$ must be updated when the measurements are made at time $t_{1}$ by propagating the change of mean through the intervening time intervals. The conditional means at time $t_{n}$ are updated again at times $t_{2}, t_{3}, \ldots, t_{n-1}$. In general, the conditional means at a given time interval must be updated whenever a measurement occurs at a previous time. In a recursive discrete-time filter with essentially an infinite number of successor time intervals, propagating the mean in this manner is not possible.

The current research [16] modifies the algorithm for updating the conditional means at subsequent times. This is done by noting that, for the discrete-time filter, the prediction of the conditional mean at all later time intervals, conditioned on the current estimate, is not necessary. For example, if the current time interval is $t_{i}$, then it is unnecessary to calculate the conditional means of $\mathbf{x}\left(t_{i+1}\right), \mathbf{x}\left(t_{i+2}\right)$, and so on. Instead of using the influence diagram algorithm for propagating the mean to these later time intervals, it is more efficient to update the mean for only the current time interval. The mean of $\mathbf{x}\left(t_{i}\right)$, given $\mathbf{x}\left(t_{i-1}\right)$, is given by the Kalman filter equation:

$$
\hat{\mathbf{x}}\left(t_{i}^{-}\right)=\boldsymbol{\Phi}\left(t_{i}, t_{i-1}\right) \hat{\mathbf{x}}\left(t_{i-1}^{+}\right) .
$$

Similarly, the mean of the measurement vector, conditioned on the current state estimate but before the actual measurement, can be calculated as

$$
\hat{\mathbf{z}}\left(t_{i}^{-}\right)=\mathbf{H}\left(t_{i}\right) \hat{\mathbf{x}}\left(t_{i}^{-}\right) \text {. }
$$

The influence diagram should still be used to calculate the conditional mean of the states at time $t_{i}$, based on the measurements at time $t_{i}$. However, when there are many successors, representing later time intervals, the conditional means for later states and measurements should be calculated only when needed using (38) and (39) above.

As in the Kalman filter, (38) can be modified to deal with deterministic inputs to the model. Such a system is described by the model:

$$
\begin{aligned}
\mathbf{x}\left(t_{i}\right)= & \boldsymbol{\Phi}\left(t_{i}, t_{i-1}\right) \mathbf{x}\left(t_{i-1}\right)+\mathbf{G}_{d}\left(t_{i-1}\right) \mathbf{w}_{d}\left(t_{i-1}\right) \\
& +\mathbf{B}_{d}\left(t_{i-1}\right) \mathbf{u}_{d}\left(t_{i-1}\right)
\end{aligned}
$$

where $\mathbf{u}_{d}\left(t_{i-1}\right)$ is the deterministic input and $\mathbf{B}_{d}\left(t_{i-1}\right)$ is the matrix that describes how the input affects the 
system states [7, p. 220]. As above, the mean of $\mathbf{x}\left(t_{i}\right)$, given $\hat{\mathbf{x}}\left(t_{i-1}^{+}\right)$and deterministic inputs, is given by the Kalman filter equation:

$$
\hat{\mathbf{x}}\left(t_{i}^{-}\right)=\boldsymbol{\Phi}\left(t_{i}, t_{i-1}\right) \hat{\mathbf{x}}\left(t_{i-1}^{+}\right)+\mathbf{B}_{d}\left(t_{i-1}\right) \mathbf{u}_{d}\left(t_{i-1}\right) .
$$

The second improvement to the influence diagram algorithm is a method of incorporating the measurements as a vector. As described earlier, the change in mean at any node is the product of the change in mean of the predecessor (measurement node) and the path coefficient between the two. Thus, propagating the mean requires calculating the path coefficients from the single realized (measured) node in the measurement vector to each successor node. For a measurement vector of $p$ nodes, and a state estimate of $n$ nodes, this process requires the calculation of the entire matrix of path coefficients $\mathbf{U}=\mathbf{U}_{1} \mathbf{U}_{2} \mathbf{U}_{3} \ldots \mathbf{U}_{n+p}$. The measurement at any node is treated as a scalar update, and the mean is propagated to all subsequent nodes.

A vector update method can be defined as follows. Assume a vector of $p$ nodes in the measurement vector as in the bottom diagram of Fig. 8. Define the residual vector as $r_{i}=\left\{\left(\zeta_{i}-\mu_{i}\right)\right.$ for $i=1$ to $\left.p\right\}$ where $\mu_{i}$ is the mean before any updates. The change in mean (the residual) and the conditional mean itself for node $p+1$, the first node of the state vector, can be calculated by

$$
r_{p+1}=b_{1, p+1} r_{1}+b_{2, p+1} r_{2}+b_{3, p+1} r_{3}+\cdots+b_{p, p+1} r_{p}
$$

$$
\zeta_{p+1}=r_{p+1}+\mu_{p+1}
$$

The new mean for node $p+1$ is treated as a measurement, and the residuals for all $p+1$ nodes can be propagated to node $p+2$, the second node of the state vector. The equation for this propagation and the resulting mean is

$$
\begin{aligned}
r_{p+2}= & \left(b_{1, p+2} r_{1}+b_{2, p+2} r_{2}+b_{3, p+2} r_{3}+\cdots+b_{p, p+2} r_{p}\right) \\
& +\left(b_{p+1, p+2} r_{p+1}\right) \\
\zeta_{p+2}= & r_{p+2}+\mu_{p+2} .
\end{aligned}
$$

The process continues with each new residual treated as a measurement and included with previous measurements to be propagated to the next node. The calculations for the last, or $n+p$ node, are

$$
\begin{aligned}
r_{p+n}= & \left(b_{1, p+n} r_{1}+b_{2, p+n} r_{2}+b_{3, p+n} r_{3}+\cdots+b_{p, p+n} r_{p}\right) \\
& +\left(b_{p+1, p+n} r_{p+1}+b_{p+2, p+n} r_{p+2} \cdots\right. \\
& \left.\quad+b_{p+n-1, p+n} r_{p+n-1}\right) \\
\zeta_{p+n}= & r_{p+n}+\mu_{p+n} .
\end{aligned}
$$

The calculation of these residuals can be treated as a series of vector/matrix products.

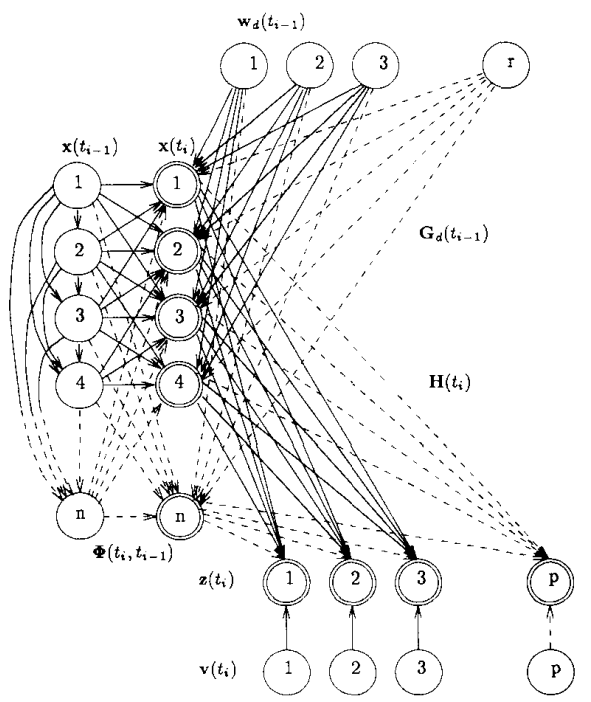

Fig. 9. One propagation/update cycle of influence diagram discrete-time filter.

This algorithm is more efficient than the earlier scalar method because it avoids the calculation of path coefficients for nodes within the measurement vector. These path coefficients are not necessary because there is no need to calculate the conditional mean for a node that already has a realized value. The total number of operations required for the vector update is $\frac{1}{2} n^{2}-\frac{1}{2} n+p n$ additions and multiplications. This can be compared with $\frac{1}{6}(n+p)^{3}-\frac{1}{2}(n+p)^{2}+\frac{1}{3}(n+p)$ additions and multiplications required to compute the $n+p$ dimensional matrix of path coefficients $\mathbf{U}$.

The third improvement to the influence diagram algorithm can be demonstrated with an alternative form of the discrete time filter. In Fig. 9, the nodes of $\mathbf{w}_{d}\left(t_{i-1}\right)$ had no arrows between them, implying they were independent. This is equivalent to assuming the matrix product $\mathbf{G}_{d}\left(t_{i-1}\right) \mathbf{Q}_{d}\left(t_{i-1}\right) \mathbf{G}_{d}^{\mathrm{T}}\left(t_{i-1}\right)$ has been factored such that $\mathbf{Q}_{d}\left(t_{i-1}\right)$ is a diagonal matrix. An alternative form of the filter is shown in Fig. 10. In this diagram, $\mathbf{w}_{d}\left(t_{i-1}\right)$ is expressed as an influence diagram factorization of the matrix product $\mathbf{G}_{d}\left(t_{i-1}\right) \mathbf{Q}_{d}\left(t_{i-1}\right) \mathbf{G}_{d}^{\mathrm{T}}\left(t_{i-1}\right)$, and $\mathbf{G}_{d}\left(t_{i-1}\right)$ is assumed to be the identity matrix. When $\mathbf{w}_{d}\left(t_{i-1}\right)$ is removed, both diagrams reduce to the same influence diagram as shown in Fig. 11.

The regression coefficients and conditional variances between the nodes of $\mathbf{x}\left(t_{i}\right)$ in Fig. 11 are the same as the regression coefficients and conditional variances for the influence diagram factorization of the matrix $\mathbf{G}_{d}\left(t_{i-1}\right) \mathbf{Q}_{d}\left(t_{i-1}\right) \mathbf{G}_{d}^{\mathrm{T}}\left(t_{i-1}\right)$ in Fig. 10. The path coefficients from $\mathbf{x}\left(t_{i-1}\right)$ to $\mathbf{x}\left(t_{i}\right)$ are unchanged from Fig. 10 to Fig. 11. But, because of the added arrows between the nodes of $\mathbf{x}\left(t_{i}\right)$, the regression coefficients from $\mathbf{x}\left(t_{i-1}\right)$ to $\mathbf{x}\left(t_{i}\right)$ must change to maintain the path coefficients. The new regression coefficients can be 


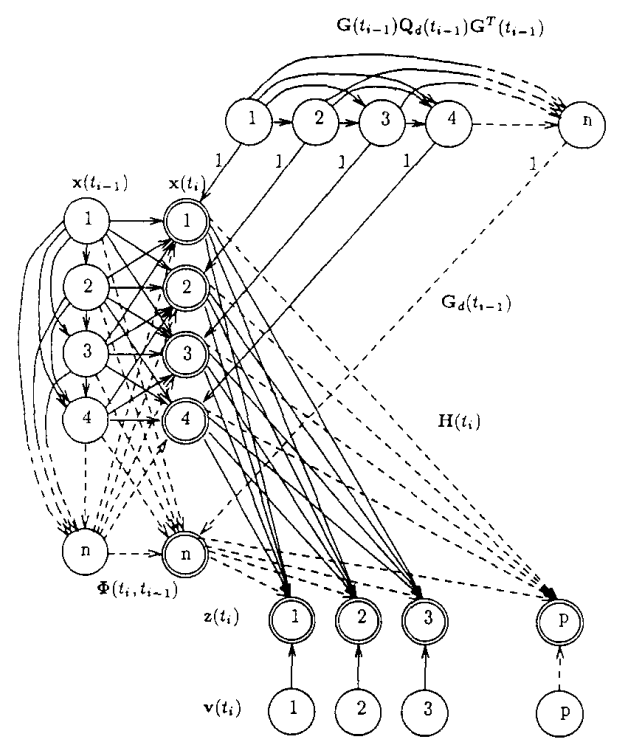

Fig. 10. Alternative representation of one propagation/update cycle of the influence diagram discrete time filter.

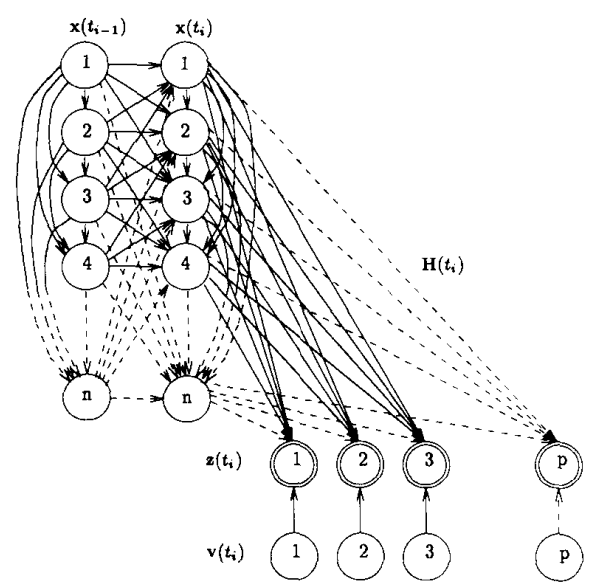

Fig. 11. Influence diagram discrete-time filter with $\mathbf{w}_{d}\left(t_{i-1}\right)$ removed.

calculated as the elements of a matrix $\boldsymbol{\Phi}_{q}$ given by

$$
\mathbf{\Phi}_{q}=\left(\mathbf{I}-\mathbf{B}_{q}\right)^{\mathrm{T}} \boldsymbol{\Phi}\left(t_{i}, t_{i-1}\right) .
$$

In (48), $\mathbf{B}_{q}$ is the influence diagram $\mathbf{B}$ matrix corresponding to the influence diagram factorization of $\mathbf{G}_{d}\left(t_{i-1}\right) \mathbf{Q}_{d}\left(t_{i-1}\right) \mathbf{G}_{d}^{\mathrm{T}}\left(t_{i-1}\right)$, and $\mathbf{I}$ is the identity matrix of appropriate dimension.

The previous analysis demonstrates a significant reduction in computations when the matrix product $\mathbf{G}_{d}\left(t_{i-1}\right) \mathbf{Q}_{d}\left(t_{i-1}\right) \mathbf{G}_{d}^{\mathrm{T}}\left(t_{i-1}\right)$ is given in influence diagram form. Under these circumstances, all influence diagram operations needed to remove $\mathrm{w}_{d}\left(t_{i-1}\right)$ are unnecessary. Instead, an influence diagram as shown in Fig. 11 can be drawn immediately, with the regression coefficients from $\mathbf{x}\left(t_{i-1}\right)$ to $\mathbf{x}\left(t_{i}\right)$ modified as in (48).
TABLE I

Operations for One Time Propagation and One Measurement Update

\begin{tabular}{|c|c|c|c|}
\hline Filter & Adds & Multiplies & Divides \\
\hline $\begin{array}{l}\text { Conven- } \\
\text { tional } \\
\text { Kalman }\end{array}$ & $\begin{array}{r}\frac{1}{2}\left(3 n^{3}+3 n^{2} p\right. \\
\quad+5 n p-n)\end{array}$ & $\begin{array}{l}\frac{1}{2}\left(3 n^{3}+3 n^{2}(p+1)\right. \\
\quad+3 n p)\end{array}$ & $m$ \\
\hline U-D & $\begin{array}{l}\frac{1}{2}\left(5 n^{3}+n^{2}(3 p+2)\right. \\
\quad+n(3 p+1))\end{array}$ & $\begin{array}{l}\frac{1}{2}\left(5 n^{3}+n^{2}(3 p+11)\right. \\
\quad+n(p-6))\end{array}$ & $n(p+1)-1$ \\
\hline $\begin{array}{l}\text { Inf luence } \\
\text { Diagram }\end{array}$ & $\begin{array}{l}2 n^{3}+n^{2}(p-0.5) \\
\quad+n\left(p^{2}+p-0.5\right)\end{array}$ & $\begin{array}{l}2 n^{3}+n^{2}(p+3.5) \\
\quad+n\left(p^{2}+5 p-1.5\right)\end{array}$ & $n(n+p-1)$ \\
\hline
\end{tabular}

Note: Assumptions: $\mathbf{G}_{d}\left(t_{i-1}\right) \mathbf{Q}_{d}\left(t_{i-1}\right) \mathbf{G}_{d}^{\mathrm{T}}\left(t_{i-1}\right)$ is available as a single matrix, for the Kalman filter, in U-D factored form for the U-D filter, or in influence diagram form for the influence diagram. State and dynamics driving noise dimension $=n$. Measurement dimension $=p$.

As mentioned earlier, all of the influence diagrams assume that the matrix $\mathbf{R}\left(t_{i}\right)$ is diagonal, as represented by the lack of arrows between the nodes of $\mathbf{v}\left(t_{i}\right)$. If $\mathbf{R}\left(t_{i}\right)$ is not diagonal, then there are two options. One option is a transformation of variables from $\mathbf{z}\left(t_{i}\right)$ to $\mathbf{z}^{*}\left(t_{i}\right)$, yielding a new $\mathbf{H}^{*}\left(t_{i}\right)$ and $\mathbf{R}^{*}\left(t_{i}\right)$ such that $\mathbf{R}^{*}\left(t_{i}\right)$ is diagonal [7, pp. 375-377]. The other option is to factor $\mathbf{R}\left(t_{i}\right)$ into influence diagram form and then reverse arcs as necessary to remove the nodes. By analogy, the influence diagram factorization of $\mathbf{R}\left(t_{i}\right)$ may be treated like the influence diagram factorization of $\mathbf{G}_{d}\left(t_{i-1}\right) \mathbf{Q}_{d}\left(t_{i-1}\right) \mathbf{G}_{d}^{\mathrm{T}}\left(t_{i-1}\right)$. All influence diagram operations to remove the nodes of $\mathbf{v}\left(t_{i}\right)$ are unnecessary because the regression coefficients and variances of $\mathbf{v}\left(t_{i}\right)$ transfer directly to $\mathbf{z}\left(t_{i}\right)$. The regression coefficients corresponding to the $\mathbf{H}\left(t_{i}\right)$ matrix are modified just as the regression coefficients corresponding to $\Phi\left(t_{i}, t_{i-1}\right)$ were modified.

\section{OPERATIONS COUNT}

Table I compares the number of mathematical operations required for the conventional Kalman filter, the U-D filter, and the influence diagram. The Kalman filter implementation assumes $\mathbf{G}_{d}\left(t_{i-1}\right) \mathbf{Q}_{d}\left(t_{i-1}\right) \mathbf{G}_{d}^{\mathrm{T}}\left(t_{i-1}\right)$ is available as a single matrix. The U-D filter implements an algorithm that uses a $\mathbf{U D U}^{\mathrm{T}}$ factored form of the covariance matrix and assumes that $\mathbf{G}_{d}\left(t_{i-1}\right) \mathbf{Q}_{d}\left(t_{i-1}\right) \mathbf{G}_{d}^{\mathrm{T}}\left(t_{i-1}\right)$ is also in $\mathbf{U D U}^{\mathrm{T}}$ form such that $\mathbf{G}_{d}$ is equal to an upper triangular $\mathbf{U}$ factor and $\mathbf{Q}_{d}$ is equal to a diagonal $\mathbf{D}$ factor. The influence diagram implementation assumes $\mathbf{G}_{d}\left(t_{i-1}\right) \mathbf{Q}_{d}\left(t_{i-1}\right) \mathbf{G}_{d}^{\mathrm{T}}\left(t_{i-1}\right)$ is expressed in influence diagram form as in Fig. 10. The only operations needed to transform the influence diagram from a form as in Fig. 10 to a form as in Fig. 11 are given in (48). The operations of (48) are included in the operations count of Table 1 .

The number of operations for the Kalman filter and the U-D filter are reproduced from Maybeck 
TABLE II

Operation Time for One Filter Recursion

\begin{tabular}{lcccc}
\hline \hline Filter & Adds & Multiplies & Divides & Time (ms) \\
\hline Conventional & & & & \\
Kalman & 1845 & 2040 & 2 & 13.36 \\
U-D & 2935 & 3330 & 29 & 21.77 \\
Influence & & & & \\
Diagram & 2205 & 2675 & 110 & 17.65 \\
\hline
\end{tabular}

Note: Assumptions: $\mathbf{G}_{d}\left(t_{i-1}\right) \mathbf{Q}_{d}\left(t_{i-1}\right) \mathbf{G}_{d}^{\mathrm{T}}\left(t_{i-1}\right)$ known as in previous table. State and dynamics driving noise dimension $=10$.

Measurement dimension $=2$. The execution time per cycle assumes each operation requires $2.7 \mu$ s per addition, $4.1 \mu$ s per multiplication, $6.6 \mu \mathrm{s}$ per division.

[7, p. 403] using the same assumptions about $\mathbf{G}_{d}\left(t_{i-1}\right) \mathbf{Q}_{d}\left(t_{i-1}\right) \mathbf{G}_{d}^{\mathrm{T}}\left(t_{i-1}\right)$ as in the previous paragraph. The matrix $\mathbf{R}\left(t_{i}\right)$ is assumed to be diagonal for all filter types. For a specific example, Table II represents the execution time for a typical discrete-time filtering problem with a 10-dimensional state vector and a 2-dimensional measurement vector. The execution times shown in Table II are the approximate single precision instruction times typical of the IBM $360[7$, p. 404]. These tables show that the influence diagram significantly exceeds the U-D filter in speed.

Under certain conditions, the matrices $\mathbf{G}_{d}\left(t_{i-1}\right) \mathbf{Q}_{d}\left(t_{i-1}\right) \mathbf{G}_{d}^{\mathrm{T}}\left(t_{i-1}\right)$ and $\boldsymbol{\Phi}\left(t_{i}, t_{i-1}\right)$ may be constant from one time interval to the next. Under these conditions, all terms on the right side of (48) are unchanged from one time interval to the next and there is no need to recompute $\boldsymbol{\Phi}_{q}$. The operations count for the influence diagram will require $n(n-1)$ fewer additions and multiplications if (48) is unnecessary. Using the assumptions of Table II, this equates to $(2.7+4.1) 45$ or 306 fewer microseconds per cycle.

\section{COMPARISON WITH MATRIX OPERATIONS}

The exchange of nodes in an influence diagram can be equated to matrix operations. For a node being made less conditional, the calculations are equivalent to computing the diagonal elements of the covariance matrix using the matrix product $\mathrm{U}^{\mathrm{T}} \mathrm{DU}$. Specifically, for the $r$ th node in an ordered sequence, $r-1$ reversals are generally needed to move the node to the beginning of the ordered sequence. The variance of this node in unconditional form can be calculated by repeated use of $(20)$ and (21). If $v_{i}$ is the original conditional variance of the $i$ th node, and $u_{i j}$ is the original path coefficient between the ith and $j$ th nodes, then the new variance of node $r$, now in unconditional form, is

$$
\begin{aligned}
P_{r r}= & v_{r}+\left(u_{r-1, r}\right)^{2} v_{r-1}+\left(u_{r-2, r}\right)^{2} v_{r-2} \\
& +\cdots+\left(u_{2, r}\right)^{2} v_{2}+\left(u_{1, r}\right)^{2} v_{1} .
\end{aligned}
$$

This is precisely the $r$ th term on the diagonal of the covariance matrix calculated by $\mathbf{U}^{\mathrm{T}} \mathbf{D U}$ related to the lower triangular Cholesky decomposition discussed earlier (this can be compared with the UDU ${ }^{\mathrm{T}}$ factorization of the U-D filter which is related to an upper triangular Cholesky decomposition). Since all terms in (49) are positive, the result must be positive. Furthermore, the unconditional variance must always be greater than or equal to the value of the conditional variance $v_{r}$.

For a node being made more conditional, the influence diagram calculations are equivalent to computing the inverse of the diagonal elements of the inverse covariance matrix using the matrix product $\left(\mathrm{U}^{\mathrm{T}} \mathrm{DU}\right)^{-1}$. For the $r$ th node in a vector of $n$ nodes, then $n-r$ reversals are needed to condition the $r$ th node on all other nodes. If $v_{r \mid r+1, r+2, \ldots, n}$ is the conditional variance of the $r$ th node conditioned on all other nodes $r+1$ through $n$, then the influence diagram computations are

$$
\begin{array}{r}
v_{r \mid r+1, r+2, \ldots, n} \\
=\frac{v_{r} v_{r+1} v_{r+2} \ldots v_{n}}{b_{r, r+1}^{2} v_{r} v_{r+2} v_{r+3} \ldots v_{n}+b_{r, r+2}^{2} v_{r} v_{r+1} v_{r+3} \ldots v_{n}} \\
+\ldots+v_{r+1} v_{r+2} v_{r+3} \ldots v_{n}
\end{array}
$$

or, if $v_{i} \neq 0$ for $i=r+1, r+2, \ldots, n$ then $v_{r \mid r+1, r+2, \ldots, n}$ can be expressed as

$$
\begin{aligned}
& \frac{1}{v_{r \mid r+1, r+2, \ldots, n}} \\
& \quad=\frac{1}{v_{r}}+\frac{b_{r, r+1}^{2}}{v_{r+1}}+\frac{b_{r, r+2}^{2}}{v_{r+2}}+\frac{b_{r, r+3}^{2}}{v_{r+3}}+\cdots+\frac{b_{r n}^{2}}{v_{n}} .
\end{aligned}
$$

The terms on the right side of (51) form the $r$ th diagonal term of the matrix product $\left(\mathbf{U}^{\mathrm{T}} \mathbf{D U}\right)^{-1}=$ $\mathbf{U}^{-1} \mathbf{D}^{-1} \mathbf{U}^{-\mathrm{T}}$ (where - T implies the inverse of the transpose). This demonstrates the relationship between the conditioning of a random variable, as calculated by the influence diagram, and the factored form of the inverse covariance matrix.

It is significant that the influence diagram algorithm can be equated to the calculation of the covariance matrix as $\mathrm{U}^{\mathrm{T}} \mathrm{DU}$, or the calculation of the inverse covariance matrix as $U^{-1} \mathbf{D}^{-1} \mathbf{U}^{-T}$. Using these relationships, current research demonstrated [16] that the influence diagram is a stable algorithm with definable error bounds. Although the demonstration is not repeated in this paper, it is similar to the method used by Wilkinson to show the error bounds for the Cholesky decomposition of a symmetric, positive definite matrix [14, p. 232].

The largest potential source of errors in the influence diagram occurs in the computation of the path coefficients $[16$, p. 118]. This is because the 
potential errors caused by the influence diagram algorithm are directly related to the errors in computing the inverse of the unit triangular matrix $\mathbf{U}=(\mathbf{I}-\mathbf{B})^{-1}$. These errors are bounded [13, p. 105] but are generally much smaller than the theoretical bounds [13, p. 106].

The operation of exchanging nodes in a covariance matrix $\mathbf{P}$ can itself be expressed in matrix form. Assume the inverse factored matrix is $\mathbf{P}^{-1}=$ $\mathbf{U}^{-1} \mathbf{D}^{-1} \mathbf{U}^{-\mathrm{T}}$ and let $\mathbf{U}^{-1}=\mathbf{V}=(\mathbf{I}-\mathbf{B})$. The factored form of the covariance matrix is $\mathbf{V}^{-\mathrm{T}} \mathbf{D V} \mathbf{V}^{-1}$. The exchange of rows and columns can be accomplished by multiplying the covariance matrix, both before and after, by a transposition matrix of the form:

$$
\mathbf{T}=\left[\begin{array}{c:cc}
\mathbf{I} & \multicolumn{2}{|c}{\mathbf{0}} \\
\hdashline \mathbf{0} & 0 & 1 \\
\hdashline & 1 & 0
\end{array}\right]
$$

where $\mathbf{T}=\mathbf{T}^{-1}$.

The new covariance matrix is TPT. In this case, $\mathbf{T}$ exchanges the $i$ th and $j$ th row and column, where $j$ is the last row of the matrix, and $i$ is the second to the last row. In general, it is possible to exchange any $i$ th and $j$ th row and column, as long as $i$ and $j$ differ by one. The new matrix, in factored form, is $\mathbf{T V}{ }^{-\mathrm{T}} \mathbf{D V} \mathbf{V}^{-1} \mathbf{T}$ or $(\mathbf{T V})^{-\mathrm{T}} \mathbf{D}(\mathbf{T V})^{-1}$. The parenthetical expression TV is no longer a triangular matrix. It can be made triangular again by postmultiplying with a matrix X such that $(\mathbf{T V X})^{-\mathrm{T}}\left(\mathbf{X}^{\mathrm{T}} \mathbf{D X}\right)(\mathbf{T V X})^{-1}=\mathbf{T P T}$. There is an $\mathbf{X}$ matrix that retriangularizes the TVX term, and maintains a diagonal middle term. One such value of $\mathbf{X}$ and $\mathbf{X}^{-1}$ that satisfies these conditions is

$$
\mathbf{X}=\left[\begin{array}{c|cc}
\mathbf{I} & \multicolumn{1}{c}{} & \mathbf{0} \\
\hdashline & b_{i j} & \frac{v_{j}}{\left(v_{j}+b_{i j}^{2} v_{i}\right)} \\
0 & 1 & \frac{-b_{i j} v_{i}}{\left(v_{j}+b_{i j}^{2} v_{i}\right)}
\end{array}\right]
$$

$$
\mathbf{X}^{-1}=\left[\begin{array}{c|cc}
\mathbf{I} & \multicolumn{2}{c}{\mathbf{0}} \\
- & -1-b_{i j} v_{i} & \frac{v_{j}}{\left(v_{j}+b_{i j}^{2} v_{i}\right)} \\
\mathbf{0} & \frac{-b_{i j}}{\left(v_{j}+b_{i j}^{2} v_{i}\right)} & 1
\end{array}\right] .
$$

After the matrix operations are carried out, the result is (TVX) ${ }^{-\mathrm{T}}\left(\mathbf{X}^{\mathrm{T}} \mathbf{D X}\right)(\mathbf{T V X})^{-1}=\mathbf{V}^{\prime-\mathrm{T}} \mathbf{D}^{\prime} \mathbf{V}^{\prime-1}$ where $\mathbf{D}^{\prime}$ is diagonal and $\mathbf{V}^{\prime}$ is upper triangular. This constitutes a reordering of the variables while still in factored form. By necessity, it must be equivalent to the factorization of the permuted matrix TPT. The expressions for $\mathbf{V}^{\prime}$ and $\mathbf{D}^{\prime}$ are:

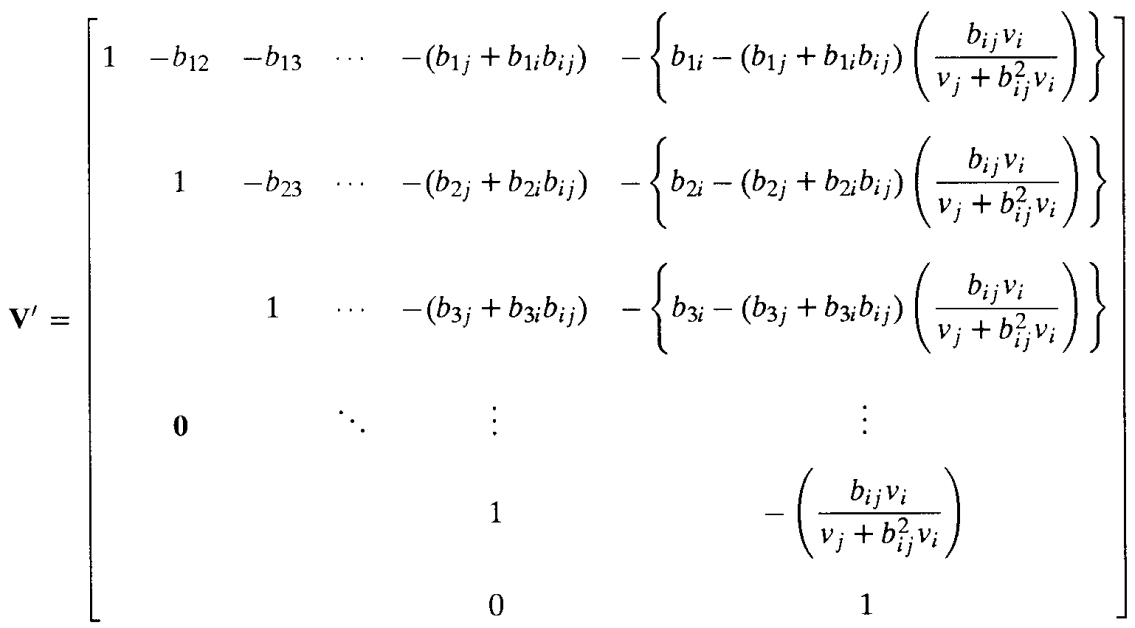

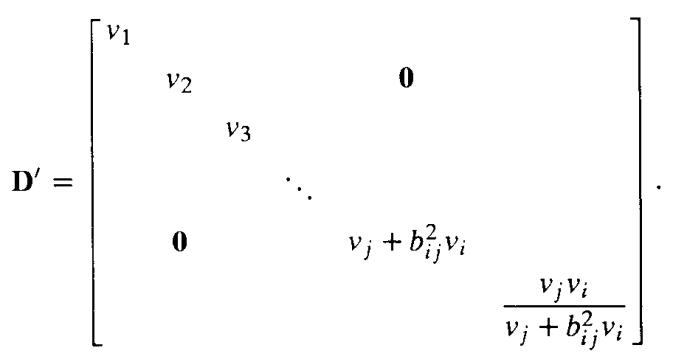


The important details of these matrices are the operations in the $i$ th and $j$ th columns. These are seen to be identical to the influence diagram operations necessary for reversing the $i$ th and $j$ th node.

\section{SUMMARY}

The influence diagram algorithm for discrete-time filtering uses a $\mathbf{U}^{\mathrm{T}} \mathbf{D U}$ factored form of the covariance matrix, analogous to the $\mathrm{UDU}^{\mathrm{T}}$ factored form of the Kalman filter discussed by Bierman $[3,6,16]$. The numerical properties of the influence diagram have not yet been extensively tested, but it has been shown that the influence diagram provides a stable algorithm. The main source of numerical errors is in the calculation of the path coefficients, where cancellation of significant digits may occur. These errors are related to the cancellation of significant digits in the computation of the inverse of a unit triangular matrix, and are very similar to the numerical errors that may occur in the U-D filter.

The influence diagram offers an alternative algorithm for discrete-time filtering. It can be faster to implement than other factored forms of the Kalman filter, while retaining numerical stability and increased precision. It can also be implemented using software packages designed for matrix computations, while the pictorial form allows insight into the meaning of the calculations.

\section{REFERENCES}

[1] Anderson, T. W. (1984) An Introduction to Multivariate Statistical Analysis. New York: Wiley, 1984.

[2] Bicrman, G. J. (1973) A comparison of discrete linear filtering algorithms. IEEE Transactions on Aerospace and Electronic Systems, AES-9, 1 (1973), 28-37.

[3] Bierman, G. J. (1977) Factorization Methods of Discrete Sequential Estimation. New York: Academic Press, 1977.
[4] Bierman, G. J., and Thornton, C. L. (1977)

Numerical comparison of Kalman filter algorithms: Orbit determination case study.

Automatica, 13 (1977), 23-35.

[5] Kaminski, P. G., et al. (1971)

Discrete square root filtering: A survey of current techniques.

IEEE Transactions on Automatic Control, AC-16, 6 (1971), 727-735.

[6] Kenley, C. R. (1986)

Influence diagram models with continuous variables. Ph.D. dissertation, Stanford University, Stanford, CA, 1986.

[7] Maybeck, P. S. (1979)

Stochastic Models, Estimation, and Control, Vol. 1.

New York: Academic Press, 1979.

[8] Mood, A. M., and Graybill, F. A. (1963) Introduction to the Theory of Statistics. New York: McGraw-Hill, 1963.

[9] Shachter, R. D. (1986)

Evaluating influence diagrams. Operations Research, 34, 6 (1986), 871-882.

[10] Shachter, R. D. (1988)

Probabilistic inference and influence diagrams. Operations Research, 36, 4 (1988), 589-604.

[11] Shachter, R. D., and Kenley, C. R. (1989) Gaussian influence diagrams. Management Science, 35, 5 (1989), 527-550.

[12] Stewart, G. W. (1973) Introduction to Matrix Computations. New York: Academic Press, 1973.

[13] Wilkinson, J. H. (1963) Rounding Errors in Algebraic Processes. Englewood Cliffs, NJ: Prentice-Hall, 1963.

[14] Wilkinson, J. H. (1965) The Algebraic Eigenvalue Problem. Oxford: Oxford University Press, 1965.

[15] Yule, G. U. (1907) On the theory of correlation for any number of variables, treated by a new system of notation. Proceedings of the Royal Society of London, (1907), 182-193; Series A.79.

[16] Zeitz, F. H. (1991)

An altemative algorithm for discrete-time filtering. M.S. thesis, Air Force Institute of Technology, Wright-Patterson Air Force Base, OH, 1991. 


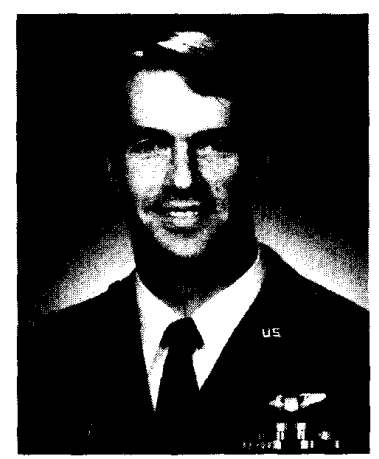

Frederick H. Zeitz, III was born on March 28, 1956 in San Antonio, TX. He received the B.S. in electrical engineering from the Air Force Academy, Colorado Springs, $C O$, in 1978, where he earned the award for being the outstanding cadet in electrical engineering. He graduated from Undergraduate Pilot Training in 1979 at Reese AFB, TX, where he remained as a T-38 instructor until February 1982. He served as Accelerated Copilot Enrichment (ACE) Assistant Detachment Commander at Minot AFB, ND as a T-38 instructor from February 1982 to April 1984. In April 1984 he attended F-111 and EF-111 qualification training at Mt. Home AFB, ID. His follow-on assignment was in the EF-111 at Upper Heyford, England assigned to the 42nd Electronic Combat Squadron. He also served as Wing Plans Officer attached to the 66th Electronic Combat Wing at Sembach AB, Germany. In May 1988, Major Zeitz was assigned to Aeronautical Systems Division (ASD) at Wright-Patterson AFB, OH and is currently the Chief of Tactical Requirements for ASD/XR. He attended the Air Force Institute of Technology as a part-time student, pursuing the M.S. degree in electrical engineering.

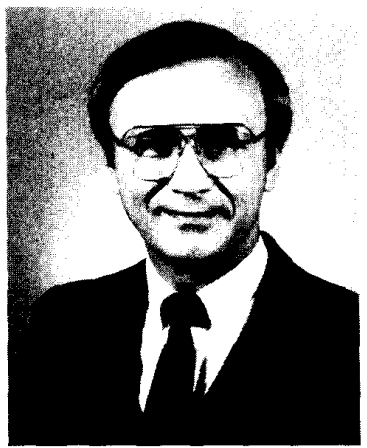

Peter S. Maybeck (S'70-M'74-SM'84-F'87) was born in New York, NY on February 9, 1947. He received the B.S. and Ph.D. degrees in aeronautical and astronautical engineering from Massachusetts Institute of Technology, Cambridge, in 1968 and 1972, respectively.

In 1968, he was employed by the Apollo Digital Autopilot Group of The C. S. Draper Laboratory, Cambridge, MA. From 1972 to 1973, he served as a military control engineer for the Air Force Flight Dynamics Laboratory and then joined the faculty of the Air Force Institute of Technology in June of 1973. He is currently Professor of Electrical Engineering, responsible for the graduate sequence in estimation and stochastic control and for individual advanced digital filtering and control courses. Current research interests concentrate on using optimal estimation techniques for guidance systems, tracking, adaptive systems and failure detection purposes.

Dr. Maybeck is a member of Tau Beta Pi, Sigma Gamma Tau, Eta Kappa Nu, and Sigma Xi. He was recipient of the DeFlorez Award (ingenuity and competence of research), the James Means Prize (excellence in systems engineering) and the Hertz Foundation Fellowship at M.I.T. in 1968. In all years from 1975 to 1992, he received commendation as outstanding Professor of Electrical Engineering at A.F.I.T. In December of 1978 , he received an award from the Affiliate Societies Council of Dayton as one of the twelve outstanding scientists in the Dayton, $\mathrm{OH}$ area. In March of 1980, he was presented with the Eta Kappa Nu Association's C. Holmes MacDonald Award, designating him as the outstanding electrical engineering professor in the United States under the age of 35 (he had placed second in this national competition for 1977 as well). In 1985, he received the Frederick Emmons Terman Award, the highest national award to a Professor of Electrical Engineering given by the American Society of Engineering Education. $\mathrm{He}$ is a member of the AIAA, and he is the current IEEE Dayton Section Student Activities Chairman and a member of the IEEE Executive Committee of Dayton, and he previously served as Chairman of the local Automatic Control Group. He is author of numerous papers on applied optimal filtering as well as the book, Stochastic Models, Estimation and Control (Academic Press, Vol. 1-1979, Vols. 2 and 3-1982). 